\title{
CONSERVAÇÃO DE POLPA DE CUPUAÇU [Theobroma grandiflorum (Willd. Ex Spreng.) Schum] POR MÉTODOS COMBINADOS ${ }^{1}$
}

\author{
MARTA CRISTINA COSTA², GERALDO ARRAES MAIA³, MEN DE SÁ MOREIRA SOUZA FILHO ${ }^{4}$, RAIMUNDO \\ WILANE DE FIGUEIREDO ${ }^{3}$, RENATA TIEKO NASSU ${ }^{4}$, JOSÉ CARLOS SABINO MONTEIRO ${ }^{3}$
}

\begin{abstract}
RESUMO - Este trabalho teve como objetivo avaliar os parâmetros químicos e físico-químicos como aporte ao desenvolvimento do processo para a conservação da polpa de cupuaçu por métodos combinados, como opção aos métodos onerosos (ex.: congelamento), como alternativa para redução de perdas pós-colheita da polpa do cupuaçu junto a pequenos e médios produtores. Foram selecionados os seguintes obstáculos: ajuste da Aw da polpa para 0,97 e 0,95, utilizando sacarose em concentrações de 22,5\% e 34\% em relação ao peso da polpa, ajuste do pH para 3,0, adição de benzoato de sódio em concentrações a $500 \mathrm{ppm}$, dióxido de enxofre $\left(\mathrm{SO}_{2}\right)$ a $400 \mathrm{ppm}$ e branqueamento a $90^{\circ} \mathrm{C} / 2$ minutos. Os resultados indicaram que os obstáculos selecionados se mostraram adequados para garantir a estabilidade microbiológica e físico-química da polpa num período de 20 dias.

Termos para indexação: Processamento, métodos combinados, cupuaçu.
\end{abstract}

\section{PRESERVATION OF CUPUAÇU PULP [Theobroma grandiflorum (Willd. Ex Spreng.)Schum] BY COMBINED METHODS}

ABSTRACT - This work had the objetive of evaluate chemical and physico-chemical standards as a support in the development of the preservation process of cupuaçu pulp by combined methods as an alternative to costly technics (ex.: freezing), reducing post-harvests lost of cupuaçu pulp for small and medium farmers. The following obstacles were chosen: water activity adjustment for values of Aw 0,97 and Aw 0,95 by addition of sucrose (22,5\% and 34\%, respectively); slow reduction of $\mathrm{pH}$ to 3,0; addition of sodium benzoate (500ppm); addition of sulfite dioxide (400ppm) and the use of branching $\left(\mathrm{T}=90^{\circ} \mathrm{C} / 2 \mathrm{~min}\right)$. The results indicated that the selected obstacles were suitable to guarantee the microbiological and physico-chemical stability of the pulp during 20 days of storage.

Index terms: Processing, combined methods, cupuaçu.

\section{INTRODUÇÃO}

A fruticultura destaca-se como um dos segmentos mais importantes da agricultura brasileira, respondendo por cerca de $25 \%$ do valor da produção agrícola nacional (Reinhardt, 1996).

O cupuaçuzeiro, Theobroma grandiflorum, é uma fruteira de grande importância para a região Amazônica. Pertence à família Sterculíaceae, gênero Theobroma. Esta espécie é encontrada espontaneamente nas áreas de mata do sul e nordeste da Amazônia oriental brasileira e nordeste do Maranhão, e também na região Amazônica de países vizinhos (Schwan, 2000). aquela que reúne as melhores condições de aproveitamento industrial (Chaar, 1980).

A alta perecibilidade, juntamente com a falta de facilidade na armazenagem durante os picos do processamento industrial contribuem para perdas pós-colheita. Deste modo, há grande expectativa para o desenvolvimento de processos para preservação local (junto ao produtor) de frutas e hortaliças por métodos combinados (Alzamora et al., 1992).

O objetivo do presente trabalho foi estudar os processos de conservação da polpa de cupuaçu por métodos combinados, tendo como obstáculos principais a atividade de água, $\mathrm{pH}$, tratamento térmico e conservantes. Esta é uma opção para a conservação da polpa, criando uma alternativa para o seu aproveitamento, visando à redução das perdas pós-colheita.

\section{MATERIALEMÉTODOS}

Frutos de cupuaçus maduros, com formato redondo, oriundos do Centro de Pesquisa Agropecuária do Trópico Úmido - CPATU/ EMBRAPA, em Belém, foram recebidos, lavados por imersão em água clorada (50ppm de cloro ativo/15min) e descascados manualmente. A polpa de cupuaçu foi obtida mecanicamente com auxílio de uma despolpadeira. Avaliou-se o rendimento em polpa. A polpa obtida foi acondicionada em sacos plásticos flexíveis de polietileno com espessura
Dentre as frutas tropicais nativas da Amazônia, o cupuaçu é

de $25 \mu$, termosselados e armazenados em congelador doméstico à temperatura de $20^{\circ} \mathrm{C}$, para análises posteriores. Inicialmente, foi realizada adição de sacarose com vistas à redução da Aw das polpas, empregando como modelo as equações de Ross (1975) e de Norrish (1966), conforme Chirife et al., (1980). Após a adequação da Aw, a polpa foi acidificada através da adição de ácido cítrico até níveis de $\mathrm{pH} 3,0$, com 1,74\% para a polpa com Aw 0,98; 1,34\% para a polpa com Aw 0,97, e 1,19\% para a polpa com Aw 0,95. Foram adicionadas às polpas as seguintes concentrações de benzoato de sódio 500, 750 e 1000ppm, visando a garantir a estabilidade microbiológica das mesmas. As polpas foram avaliadas por meio de determinações de bolores, leveduras e contagem total de microrganismos aeróbios mesófilos. Utilizaram-se 200 e 400 ppm de $\mathrm{SO}_{2}$ com o intuito de avaliar o efeito protetor quanto ao escurecimento da polpa, sendo também testado o efeito do tempo e da temperatura na operação de branqueamento $\left(90^{\circ} \mathrm{C}\right.$ por 1 e 2 minutos $)$ frente à eficiência na redução da carga microbiana da polpa e na alteração da cor da polpa. Em seguida, a polpa foi resfriada e armazenada à temperatura ambiente $\left(25^{\circ} \mathrm{C}\right)$.

O fluxograma do processamento de polpa conservada por métodos combinados está demonstrado na Figura 1.

Com o objetivo de avaliar as melhores aplicações dos obstáculos para a conservação da polpa do cupuaçu, vinte quilos de polpa foram obtidos e, em seguida, divididos em amostras de $250 \mathrm{~g}$. Para as análises físico-químicas e microbiológicas efetuadas a cada quatro dias por um período de 20 dias, foram tomadas três amostras e as análises realizadas em triplicata. A cor foi determinada segundo Hunter (1975). As determinações de sólidos solúveis totais, $\mathrm{pH}$ e dióxido de enxofre foram efetuadas de acordo com a Association of Official Agriculture Chemists (1992) e acidez total titulável (ATT) de acordo com o Instituto Adolfo Lutz (1985). Os açúcares redutores e totais, conforme Miller (1959). A atividade de água foi medida diretamente no medidor Aqualab, modelo CX-2.

As determinações microbiológicas de contagem total de microrganismos aeróbios mesófilos, bolores, leveduras e coliformes foram realizadas segundo a American Public Health Association (2001); Silva, et al. (2001).

\footnotetext{
${ }^{1}$ (Trabalho 099/2002). Recebido: 14/06/2002. Aceito para publicação: 04/07/2003.

${ }^{2}$ Bolsista da Capes.

${ }^{3}$ Departamento de Tecnologia de Alimentos/UFC Cx. Postal 12168, E-mail: frutos@ufc.br.Fortaleza-Ceará, Brasil

${ }^{4}$ Empresa Brasileira de Pesquisa Agropecuária/EMBRAPA, Rua Dra. Sara Mesquita, 2270. Planalto Pici. CEP 60511-110 - Fortaleza-CE. Fax: (0xx85) 2991833.
} 


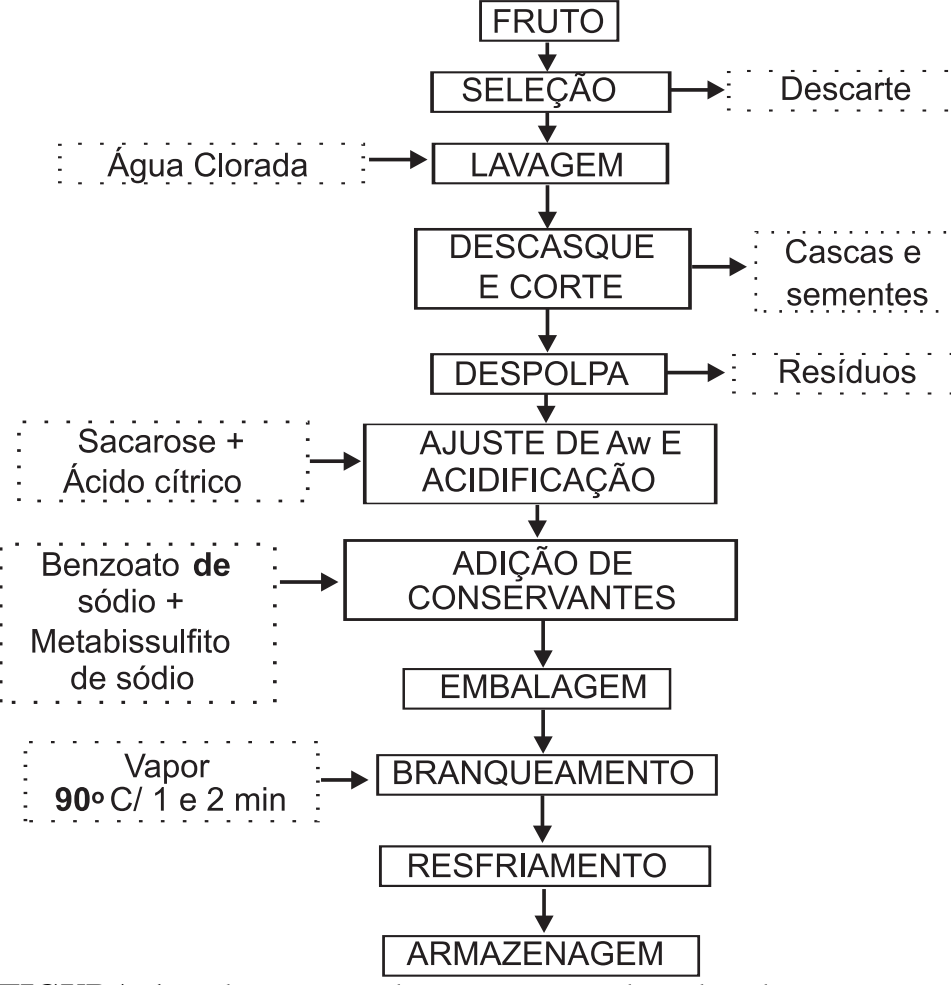

FIGURA 1 - Fluxograma de conservação da polpa de cupuaçu por processamento mínimo/métodos combinados com emprego da tecnologia de obstáculos.

\section{RESULTADOSE DISCUSSÃO}

A polpa de cupuaçu apresentou valores para mofos e leveduras de $1,1 \times 10^{2} \mathrm{UFC} / \mathrm{g}$ e para contagem padrão em placa de 5,5 x $10^{2} \mathrm{UFC} / \mathrm{g}$. Estes resultados evidenciaram a qualidade microbiológica satisfatória de acordo com os atuais padrões estabelecidos pela Agência Nacional de Vigilância Sanitária (2001). A polpa de cupuaçu apresentou as características químicas e físico-químicas médias de $\mathrm{pH} 3,34{ }^{\circ}{ }^{\circ}$ Brix 12,5; ATT 2,27\%, e Aw 0,98. Estes resultados mostram que a polpa estava dentro dos padrões de identidade e qualidade (PIQs) estabelecidos para a polpa de cupuaçu segundo o Ministério da Agricultura e do Abastecimento (2000), onde os valores mínimos estabelecidos foram mantidos. Na adequação da atividade de água, chegou-se a valores de 0,95 e 0,97, sendo necessária a adição de sacarose de $22,5 \%$ e $34 \%$, respectivamente. A Figura 2 ilustra o efeito da adição de sacarose na atividade de água da polpa de cupuaçu. Visando ao ajuste do $\mathrm{pH}$, foram necessárias dosagens de ácido cítrico de $1,74 \%$ para a polpa com Aw 0,$98 ; 1,34 \%$ para polpa com Aw 0,97, e $1,19 \%$ para polpa com Aw 0,95, para a redução do $\mathrm{pH}$ para o nível de 3,0. A Figura 3 mostra o gráfico e as equações da curva de $\mathrm{pH}$ x ácido cítrico adicionado na polpa de cupuaçu. Os valores de ATT $(\%)$ da polpa de cupuaçu, para as diferentes dosagens de ácido cítrico adicionadas, podem ser demonstrados através da Figura 4. A polpa corrigida para pH 3,0 e com Aw 0,97 e Aw 0,95 apresentou valores finais de ${ }^{\circ}$ Brix de 35,64 e 44,10 e ATT(\%) de 3,13\% e 2,66\%, respectivamente. Nas condições descritas, foi possível ajustar $\mathrm{Aw}$ e $\mathrm{pH}$ para a faixa adequada na conservação de frutos por métodos combinados. Com relação ao efeito do benzoato de sódio, em todas as concentrações empregadas, para a polpa com $\mathrm{pH} 3,0$ e pH 3,5 e Aw ajustadas, verificaram-se, para as amostras de polpa com Aw 0,98 e para as amostras com Aw 0,95 e 0,97 , valores de bolores e leveduras entre 1,5x $10^{1}$ $\mathrm{UFC} / \mathrm{g}$ e $7,5 \mathrm{x} 10^{1} \mathrm{UFC} / \mathrm{g}$ e contagem padrão entre $1 \times 10^{1} \mathrm{UFC} / \mathrm{g}$ e $5,5 \times 10^{1}$ $\mathrm{UFC} / \mathrm{g}$, após sete dias de armazenagem à temperatura ambiente. A polpa sem nenhum tratamento apresentou valores de $2,3 \times 10^{8} \mathrm{UFC} / \mathrm{g}$ para bolores e leveduras e $5,5 \times 10^{2} \mathrm{UFC/g}$ na contagem padrão no mesmo período. Os valores de $\mathrm{L}^{*}$ do acompanhamento do escurecimento da polpa em que se verificou maior tendência ao escurecimento (menores valores de L) para as amostras, foram aqueles com menor atividade de água. Esta tendência ao escurecimento pode ser associada à incorporação de sacarose para ajuste de Aw, que, por influência da acidez e baixo $\mathrm{pH}$, tem favorecida sua hidrólise no decorrer da armazenagem, elevando a concentração de grupos redutores e criando condições adequadas ao surgimento de escurecimento não-enzimático (Tabela 1). Verificou-se maior tendência ao escurecimento nas amostras sem adição e com adição de 200ppm de $\mathrm{SO}_{2}$, enquanto as amostras com 400ppm de $\mathrm{SO}_{2}$ apresentaram menor tendência, mantendo boa estabilidade de sua coloração, o que pode ser observado pelo maior valor de $\mathrm{L}$ nas amostras com 400ppm de $\mathrm{SO}_{2}$ durante os 20 dias de armazenagem. Na Tabela 2 , são apresentados os valores residuais de $\mathrm{SO}_{2}$ em polpa de cupuaçu com diferentes níveis de Aw, durante a armazenagem de 20 dias. Os resultados referentes ao efeito do branqueamento são apresentados nas Tabelas 3 e 4 , onde se verifica que o tratamento por dois minutos a $90^{\circ} \mathrm{C}$ ofereceu melhor resposta quanto aos atributos de cor e estabilidade microbiológica.

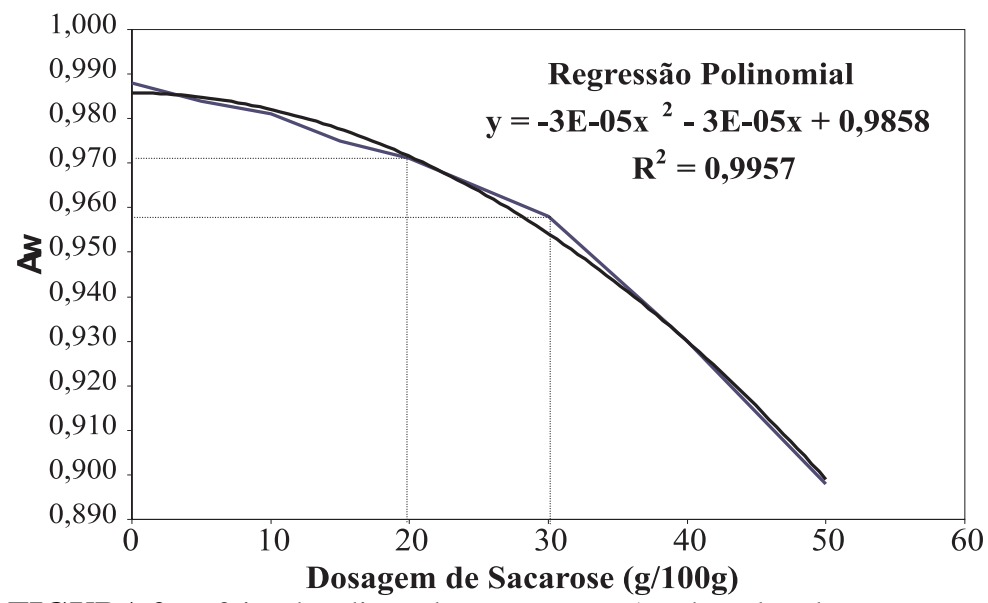

FIGURA 2 - Efeito da adição de sacarose na Aw da polpa de cupuaçu.

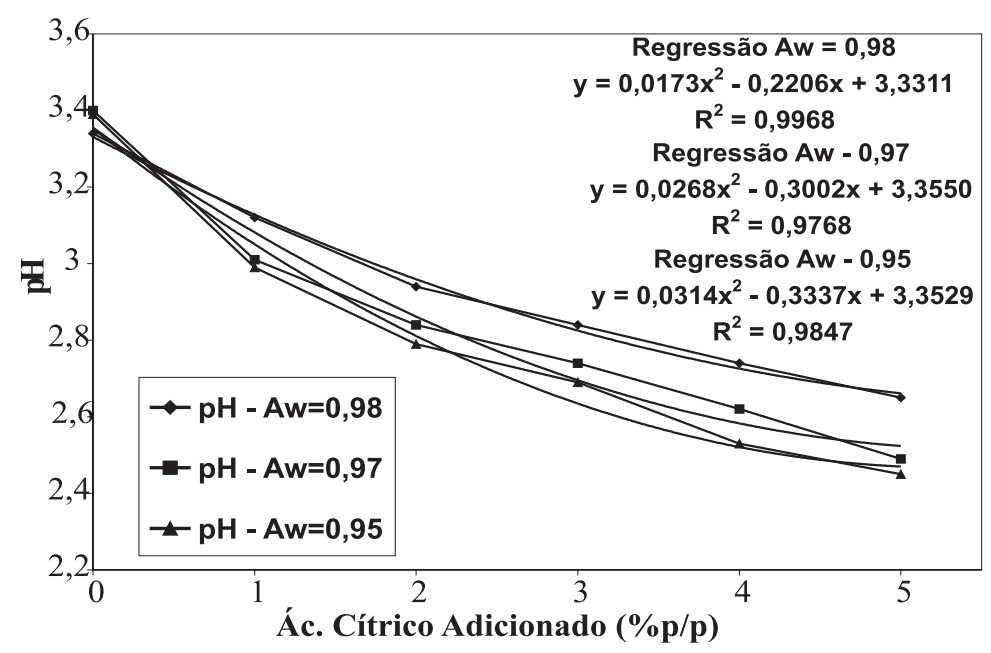

FIGURA 3 - Efeito da adição de ácido cítrico no pH da polpa de cupuaçu.

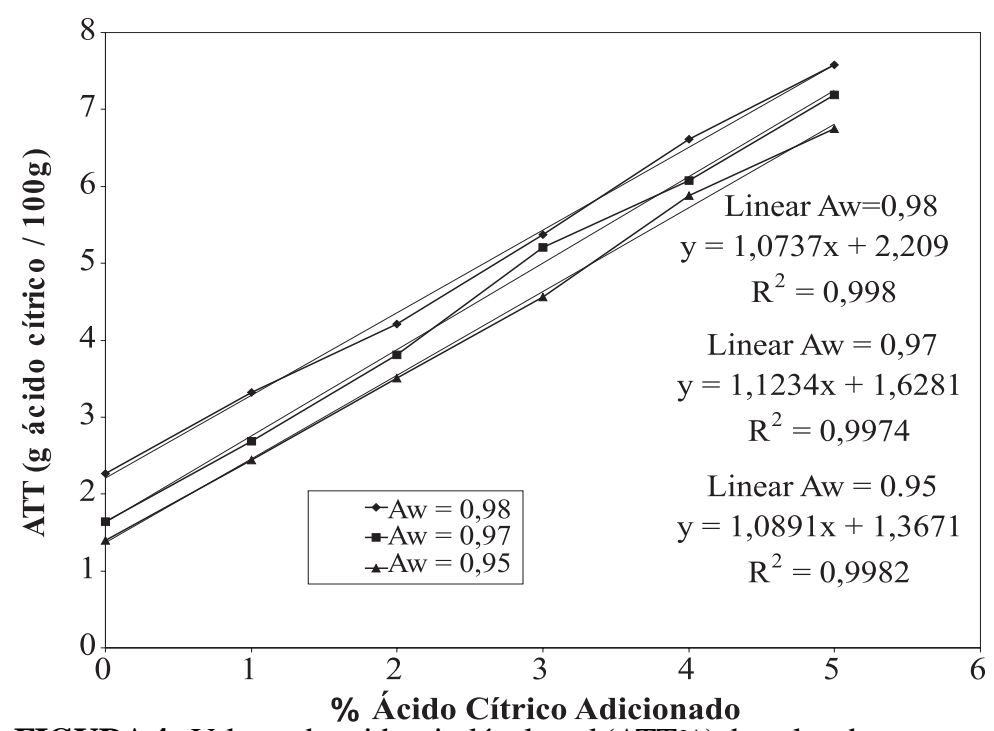

FIGURA 4 - Valores de acidez titulável total (ATT\%) da polpa de cupuaçu para as diferentes dosagens de ácido cítrico. 
TABELA 1 - Valores de L durante a armazenagem da polpa de cupuaçu com diferentes níveis de atividade de água e concentrações de $\mathrm{SO}_{2}$.

\begin{tabular}{|c|c|c|c|c|c|c|c|c|c|}
\hline \multirow{3}{*}{$\begin{array}{c}\text { Tempo } \\
\text { (dias) }\end{array}$} & \multicolumn{9}{|c|}{ Amostras } \\
\hline & \multicolumn{3}{|c|}{ Aw 0,98 } & \multicolumn{3}{|c|}{ Aw $\quad 0,97$} & \multicolumn{3}{|c|}{ Aw 0,95} \\
\hline & $\mathrm{Sem} \mathrm{SO}_{2}$ & $200 \mathrm{ppm}$ & 400 ppm & $\mathrm{Sem} \mathrm{SO}_{2}$ & $200 \mathrm{ppm}$ & 400 ppm & $\mathrm{Sem} \mathrm{SO}_{2}$ & $200 \mathrm{ppm}$ & 400 ppm \\
\hline 0 & 63,25 & 63,57 & 63,63 & 53,27 & 51,90 & 54,73 & 46,47 & 46,43 & 46,27 \\
\hline 4 & 57,20 & 63,20 & 63,90 & 48,07 & 52,53 & 53,23 & 41,50 & 45,83 & 45,40 \\
\hline 8 & 56,40 & 60,85 & 63,97 & 46,23 & 50,13 & 52,73 & 40,37 & 44,93 & 45,17 \\
\hline 12 & 56,86 & 58,40 & 61,57 & 45,57 & 49,10 & 52,57 & 39,20 & 43,17 & 45,47 \\
\hline 16 & 59,07 & 56,97 & 60,23 & 44,73 & 48,30 & 51,90 & 38,70 & 42,57 & 44,90 \\
\hline 20 & 57,27 & 56,17 & 64,33 & 45,70 & 47,30 & 53,87 & 38,10 & 43,43 & 46,70 \\
\hline
\end{tabular}

TABELA 2 - Efeito da atividade de água no decréscimo de $\mathrm{SO}_{2}$ em polpa de cupuaçu com os níveis de $\mathrm{Aw}$, com $400 \mathrm{ppm}$ de $\mathrm{SO}_{2}$ inicial, mantida à temperatura ambiente $\left(25^{\circ} \mathrm{C}\right)$.

\begin{tabular}{c|ccc}
\hline \multirow{2}{*}{ Tempos (dias) } & \multicolumn{3}{|c}{ Residual de $\mathbf{S O}_{\mathbf{2}} \mathbf{( \% )}$} \\
\cline { 2 - 4 } & \multicolumn{3}{|c}{ Atividade de Água } \\
\cline { 2 - 4 } & Aw 0,98 & Aw 0,97 & Aw 0,95 \\
\hline 0 & 100,00 & 100,00 & 100,00 \\
4 & 98,92 & 94,64 & 85,77 \\
8 & 66,56 & 69,64 & 65,26 \\
12 & 34,59 & 45,80 & 44,56 \\
16 & 27,53 & 46,05 & 39,78 \\
20 & 19,21 & 28,56 & 35,81 \\
\hline
\end{tabular}

TABELA 3 - Efeito do branqueamento na cor das polpas de cupuaçu nos diferentes tempos de exposição $(0,1$ e 2 min)

\begin{tabular}{|c|c|c|c|c|c|c|c|c|c|}
\hline \multirow{3}{*}{ Tempo } & \multicolumn{9}{|c|}{ Determinações de cor $(\mathrm{L})$} \\
\hline & \multicolumn{3}{|c|}{ Aw 0,98} & \multicolumn{3}{|c|}{ Aw 0,97} & \multicolumn{3}{|c|}{ Aw 0,95} \\
\hline & $\mathbf{L}$ & $\mathbf{A}$ & B & $\mathbf{L}$ & $\mathbf{a}$ & B & $\mathbf{L}$ & $\mathbf{a}$ & b \\
\hline $0 \min$ & 67,98 & $-2,17$ & 27,27 & 58,71 & $-2,94$ & 24,39 & 52,97 & $-3,32$ & 19,51 \\
\hline 0 min & 68,56 & $-2,77$ & 26,7 & 59,2 & $-3,11$ & 24,37 & 52,56 & $-2,95$ & 19,21 \\
\hline $0 \mathrm{~min}$ & 68,23 & $-2,42$ & 27,55 & 59,45 & $-3,4$ & 23,87 & 52,9 & $-2,72$ & 20,23 \\
\hline $1 \mathrm{~min}$ & 68,20 & $-2,42$ & 27,55 & 58,48 & $-3,05$ & 23,8 & 52,14 & $-3,41$ & 19,76 \\
\hline $1 \mathrm{~min}$ & 68,70 & $-2,54$ & 27,94 & 58,4 & $-3,38$ & 23,6 & 51,84 & $-3,26$ & 19,36 \\
\hline 1 min & 68,13 & $-2,31$ & 27,81 & 58,03 & $-3,38$ & 23,11 & 50,58 & $-2,97$ & 18,3 \\
\hline $2 \mathrm{~min}$ & 66,41 & $-2,79$ & 26,38 & 57,05 & $-3,05$ & 22,8 & 51,52 & $-2,92$ & 19,08 \\
\hline $2 \min$ & 66,68 & $-2,44$ & 28,74 & 58,01 & $-3,27$ & 23,69 & 52,23 & $-2,79$ & 20,22 \\
\hline $2 \min$ & 66,04 & $-2,95$ & 26,8 & 58,34 & $-2,92$ & 24,32 & 51,61 & $-2,97$ & 19,85 \\
\hline
\end{tabular}

TABELA 4 - Efeito do branqueamento na estabilidade microbiológica da polpa de cupuaçu à temperatura de $90^{\circ} \mathrm{C}$ por 2 min.

\begin{tabular}{l|c|c|c|c|c}
\hline \multirow{2}{*}{ Amostras } & \multicolumn{5}{|c}{ Determinações Microbiológicas } \\
\cline { 2 - 6 } & $\begin{array}{c}\text { Contagem padrão } \\
\text { (UFC/g) }\end{array}$ & $\begin{array}{c}\text { Mofos e } \\
\text { leveduras } \\
\text { (UFC/g) }\end{array}$ & \multicolumn{3}{|c}{ Coliformes (NMP/g) } \\
\cline { 3 - 6 } & & $\mathbf{3 5}^{\circ} \mathbf{C}$ & $\mathbf{4 5}^{\circ} \mathbf{C}$ & E. coli \\
\hline Aw 0,98 & $1,6 \times 10^{2}$ & $8,5 \times 10^{2}$ & $<3$ & $<3$ & $<3$ \\
Aw 0,97 & $1,4 \times 10^{2}$ & $2,2 \times 10^{2}$ & $<3$ & $<3$ & $<3$ \\
Aw 0,95 & $4,2 \times 10^{1}$ & $1,3 \times 10^{3}$ & $<3$ & $<3$ & $<3$ \\
\hline
\end{tabular}

\section{CONCLUSÕES}

Os obstáculos selecionados: pH 3,0; Aw 0,98; Aw 0,97, e Aw 0,95, 500ppm de benzoato de sódio e 400ppm de $\mathrm{SO}_{2}$, foram adequados e eficientes para manter o controle microbiológico e controlar o escurecimento da polpa de cupuaçu durante um período de 20 dias de armazenamento à temperatura ambiente $\left(25^{\circ} \mathrm{C}\right)$.

\section{REFERÊNCIASBIBLIOGRÁFICAS}

AGÊNCIA NACIONAL DE VIGILÂNCIA SANITÁRIA. Resolução n ${ }^{\circ} 12$ de 02 de fevereiro de 2001. Estabelece padrões microbiológicos para alimentos.

ALZAMORA, S.M.; ARGAIZ, A., WELTI,J. Fruit preservation by combined factors. Food Research International, Oxford, v.25, n. 2, p.159$165,1992$.

AMERICAN PUBLIC HEALTHASSOCIATION. Compendium of methods for the microbiological examination of foods. 4. ed. Washington, DC: American public health association, 2001, 676p.

A O A C. Oficial Methods of Analysis of the Association of Oficial Analytical Chemistry. 12 ed. Washington, 1992.

CHAAR, J.M. Composição do cupuaçu (Theobroma grandiflorum Schum) e conservação de seu néctar por meios físicos e químicos. Rio de Janeiro, 1980, 78p. Tese Mestrado - Univ. Fed. Rural do Rio de Janeiro.

CHIRIFE, J.; FERRO FONTAN, C.; BENMERGUI, E. A. The prediction of water activity of aqueous solutions in connection with intermediate moisture foods IV. Aw prediction in aqueous non-electrolyte solution. Journal of Food Technology, v.15, n. 59, 1980.

HUNTER, R. S. The instruments of appearence. New York: John Wiley and Son, 1975.

INSTITUTO ADOLFO LUTZ. Normas Analíticas do Instituto Adolfo Lutz: métodos químico e físicos para análises de alimentos. 3.ed. São Paulo, 1985, v.1, 533p.

MILLER,G.L. Use of dinitrosalicycle acid reagent for determination of reducing sugars. Analytical Chemistry, Washington, n.31, p. 26$248,1959$.

MINISTÉRIO DAAGRICULTURAEDO ABASTECIMENTO. Instrução Normativa ${ }^{\circ} 1$ de 07 de janeiro de 2000. Aprova o Regulamento Técnico para fixação dos Padrões de Identidade e Qualidade para polpa de fruta. Diário Oficial da União, Brasília-DF,Seção I, p.54.

NORRISH, R. S. An equation for the activity coeficients and equilibrium relative humidities of water in confectionerv syrups. Journal Food Technology, v.1, p. 25-39, 1966.

REINHARDT, D.H. Avanços tecnológicos na fruticultura tropical. Informativo da Sociedade Brasileira de Fruticultura. Brasília: v.15, n.4, p.18-21, dez. 1996.

ROSS, K.D. Estimation of water activity in intermediate moisture foods. Food Technology, Chicago, v.29, n.3, p.26-34, 1975.

SCHWAN, R. F. et al. Cupuaçu [Theobroma grandiflorum (Willd Ex Spreng.)]. In: ALVES, R. E., FILGUEIRAS, H.A.C., MOURA, C.F.H. (coords.). Caracterização de frutas nativas da América Latina. Jaboticabal: FUNEP, 2000. P.31-34 (Série Frutas Nativas, 9).

SILVA, N. et al. Manual de Métodos de Análise Microbiológica de Alimentos. 2.ed. Livraria Varela. São Paulo, 2001. 229p. 